\title{
Effects of volatile additives in solutions used to prepare polythiophene-based thin-film transistors
}

\author{
Musubu Ichikawa, ${ }^{1,2, a)}$ Kenta Yamamura, ${ }^{1}$ Hyeon-Gu Jeon, ${ }^{1}$ Miyako Nakajima, ${ }^{1}$ \\ and Yoshio Taniguchi ${ }^{3}$ \\ ${ }^{1}$ Functional Polymer Science Course, Division of Chemistry and Materials, Faculty of Textile Science \\ and Technology, Shinshu University, 3-15-1 Tokida, Ueda 386-8567, Japan \\ ${ }^{2}$ PRESTO, Japan Science and Technology Agency (JST), 4-8-1 Honcho, Kawaguchi, Saitama 332-0012, Japan \\ ${ }^{3}$ Shinshu University, 3-15-1 Tokida, Ueda 386-8567, Japan
}

(Received 15 November 2010; accepted 8 January 2011; published online 10 March 2011)

\begin{abstract}
We investigate the effects of volatile additives in solutions used to prepare thin-film transistors (TFTs) of regioregular poly(3-hexylthiophene) (P3HT). We use the additives trifluoromethylbenzene (TFMB) and methylcyclohexane (MCH) because they are poor solvents for P3HT. The additives improve the performance of the resulting TFTs when the boiling point $\left(T_{\mathrm{b}}\right)$ of the major solvent, carbon tetrachloride, is lower than that of the additive. The maximum mobility is $(4.0 \pm 0.9) \times 10^{-2}$ $\mathrm{cm}^{2} \mathrm{~V}^{-1} \mathrm{~s}^{-1}$, which is 6.1 times larger than that of TFTs prepared without TFMB or MCH added to the solution; the on/off ratio and the subthreshold slope were also improved. The relative $T_{\mathrm{b}}$ of the solvent and the additive affected the film formation with the amount of TFMB or MCH remaining at the final stage of thin film deposition influencing the precipitation of P3HT aggregates. (C) 2011 American Institute of Physics. [doi:10.1063/1.3553878]
\end{abstract}

\section{INTRODUCTION}

Organic thin-film transistors (TFTs) have received much attention because of their potential applications in flat panel displays, flexible electronic devices, and low-cost identification tags. Small molecule-based organic TFTs fabricated by vacuum evaporation techniques have already surpassed hydrogen-terminated amorphous silicon $(\alpha-\mathrm{Si}: \mathrm{H})$-based TFTs from the viewpoint of field-effect mobility $\left(\mu_{\mathrm{FET}}\right)$; for example, $\mu_{\mathrm{FET}}$ is $5.5 \mathrm{~cm}^{2} \mathrm{~V}^{-1} \mathrm{~s}^{-1}$ for a pentacene-based p-type TFT; ${ }^{1}$ $2.9 \mathrm{~cm}^{2} \mathrm{~V}^{-1} \mathrm{~s}^{-1}$ for a dinaphtho[2,3-b:2', $3^{\prime}$-f]thieno[3,2-b]thiophene-based p-type TFT; $2.1 \mathrm{~cm}^{2} \mathrm{~V}^{-1} \mathrm{~s}^{-1}$ for a perylene diimide-based n-type TFT; ${ }^{3}$ and $6 \mathrm{~cm}^{2} \mathrm{~V}^{-1} \mathrm{~s}^{-1}$ for a $\mathrm{C}_{60}$ fullerenebased n-type TFT. ${ }^{4}$ Organic TFTs based on polymers have also attracted interest because their production is cost-effective, energy efficient, and applicable to large areas, making them suitable for future printable electronics.

Regioregular poly(3-hexylthiophene) (P3HT), which was first reported by Sirringhaus et al. in $1999,{ }^{5}$ is a promising candidate as a semiconducting material for polymer TFTs. ${ }^{6}$ The high regioregularity of P3HT allows it to form highly crystallized semiconducting thin films; this leads to high hole $\mu_{\mathrm{FET}}{ }^{5,7}$ Methods for obtaining highly organized thin films include annealing thin films after preparation, ${ }^{8}$ using highboiling-point solvents, ${ }^{9}$ and preparing highly concentrated solutions. ${ }^{10}$ Recently, Ong et al. ${ }^{11}$ reported that charge transfer (CT) from an analog of P3HT [poly $\left(3,3^{\prime \prime \prime}\right.$-didodecylquaterthiophene), PQT-12] to the slightly electron-accepting dichlorobenzene caused the formation of a lamellar structure. $\mathrm{CT}$ induces coplanar structures in thiophene-based polymers because $\mathrm{CT}$ states of polythiophene act as polarons with a quinoid structure. ${ }^{12}$ In this context, chemical doping to influence

\footnotetext{
${ }^{\text {a) }}$ Author to whom correspondence should be addressed. Electronic mail: musubu@shinshu-u.ac.jp.
}

CT interactions appears to be useful for forming highly ordered thin films based on polythiophene because the addition of a dopant is a simple means for achieving polymer TFTs with better performance. However, the effect of the remaining dopants should be considered because the remaining CT-type dopants probably increase the conductivity of the thin films and then reduce the on/off ratio of PHT TFTs. In fact, traces of metal impurities in P3HT strongly reduce the on/off ratio of P3HT TFTs. ${ }^{13}$

However, if the additives are highly volatile, it can be assumed that the remaining additives do not cause a negative effect. In addition, evaporation of the solvent should accompany the formation of polymer thin films from solution; hence, this process does not occur at equilibrium. Therefore volatile additives in polymer solutions should potentially affect the resulting thin films. Here we investigate the effects of adding volatile compounds to P3HT solutions. The addition of a dopant improves the TFT performance because the dopant helps in the formation of highly ordered thin films. We find that adding volatile dopants enhances the mobility, on/off ratio, and subthreshold slope of P3HT TFTs.

\section{EXPERIMENTAL}

Figure 1 shows the device structure used in this study. The substrates were a $\mathrm{n}^{2+}-\mathrm{Si}$ wafer (gate electrode) with a thermally grown 200-nm-thick $\mathrm{SiO}_{2}$ layer (gate insulator). The Tokyo Sanyo vacuum deposited a 150 -nm-thick indium tin oxide (ITO) layer on the substrates. To create source and drain electrodes, the ITO layers were patterned by photolithography, a wet-etching technique with photoresist (Tokyo Ohka Kogyo THMR-iP 3300), and a commercial ITO etchant (Kanto Chemicals ITO-02). The length and width of the channels were $30 \mu \mathrm{m}$ and $2 \mathrm{~mm}$, respectively. ITO functions as a suitable contact electrode for organic TFTs in bottom-contact 

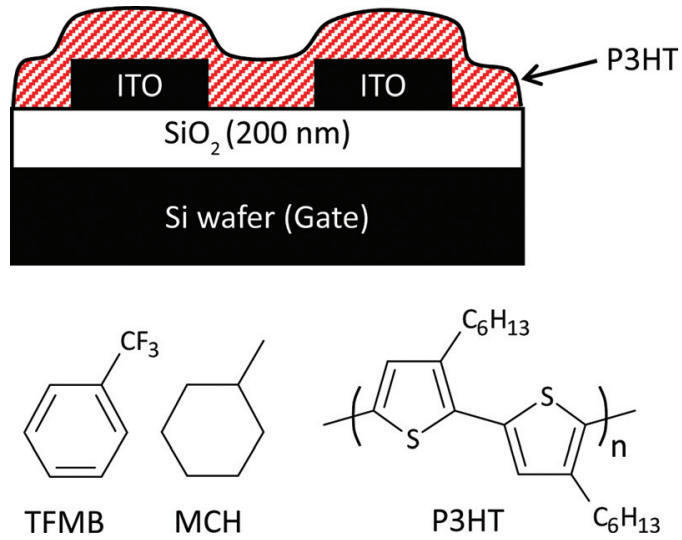

FIG. 1. (Color online) Device structure (top) and chemical structures of volatile additives and P3HT (bottom). The thickness of the P3HT layer depended on the solvent used and it ranged from about 100-160 nm.

configurations from the viewpoint of contact resistance. ${ }^{14}$ Prior to preparing $\mathrm{P} 3 \mathrm{HT}$ layers on the substrates, the substrate surfaces were modified by exposing the substrates to $\mathrm{O}_{2}$ plasma for $5 \mathrm{~min}$ and then treating them with a toluene solution containing $0.1 \mathrm{wt}$. \% octyltrichlorosilane in a $\mathrm{N}_{2}$-filled glove box for $30 \mathrm{~min}$ at $70^{\circ} \mathrm{C}$. The treated substrates were rinsed with absolute toluene and 2-propanol sequentially and then sonicated in 2-propanol for 30 min. P3HT (Aldrich) was purified before use by Soxhlet extraction with acetone for $12 \mathrm{~h}$, methanol for $6 \mathrm{~h}$, and hexane for $30 \mathrm{~h}$. P3HT solutions (1 wt. \%) were prepared in carbon tetrachloride (CTC), $p$-xylene, toluene, tetrahydrofuran (THF), chloroform, and $o$-dichlorobenzene (DCB). Volatile additives [trifluoromethylbenzene (TFMB) or methylcyclohexane $(\mathrm{MCH})$, Fig. 1] were added at certain concentrations. The concentration of the volatile additives in the final solutions varied from 0 to $20 \mathrm{vol}$. \%. The final solutions were filtered through poly (tetrafluoroethylene) membranes with $0.2-\mu$ m-diameter pores. Thin films of P3HT were fabricated on the Si substrates by spin coating at $2500 \mathrm{rpm}$ for $60 \mathrm{~s}$ and then drying in a vacuum oven at $100^{\circ} \mathrm{C}$ for $1 \mathrm{~h}$. The characteristics of P3HT TFTs were evaluated in a vacuum chamber in the dark using a semiconductor device analyzer (Agilent B1500A) with three high power source meters (Agilent HPSMU). X-ray diffraction and topographical observations of the $\mathrm{P} 3 \mathrm{HT}$ thin films were carried out using a Rigaku rotaflex diffractometer with $\mathrm{Cu}-\mathrm{K} \alpha$ radiation (154 pm) and SII nanotechnology SPA-400 atomic force microscope (AFM) in dynamic force mode, respectively. Note that the same samples were used for TFT measurements and for performing these evaluations. The thickness of the thin films was verified using a Tencor Alpha-Step 500 profilometer. Table I shows several properties of the solvents used in his study.

\section{RESULTS AND DISCUSSION}

\section{A. Effect of TFMB addition to P3HT in CTC}

Figure 2 shows the drain current $\left(I_{\mathrm{D}}\right)$-drain voltage $\left(V_{\mathrm{D}}\right)$ and drain current $\left(I_{\mathrm{D}}\right)$-gate voltage $\left(V_{\mathrm{G}}\right)$ characteristics of ${ }^{\circ} \mathrm{CP} 3 \mathrm{HT}$ TFTs prepared from CTC solutions with (CTC: TFMB $=10: 1)$ and without the volatile additive TFMB. Both TFTs worked as p-type transistors. The addition of
TABLE I. Selected properties of solvents and additives.

\begin{tabular}{lccccc}
\hline \hline & $\begin{array}{c}\text { Boiling } \\
\text { point } \\
\left({ }^{\circ} \mathrm{C}\right)\end{array}$ & $\begin{array}{c}\text { Melting } \\
\text { point } \\
\left({ }^{\circ} \mathrm{C}\right)\end{array}$ & $\begin{array}{c}\text { Density } \\
\text { at } 20^{\circ} \mathrm{C} \\
(\mathrm{g} / \mathrm{ml})\end{array}$ & $\begin{array}{c}\text { Specific } \\
\text { permittivity }\end{array}$ & $\begin{array}{c}\text { Solubility of } \\
\text { P3HT at } \\
\left.40^{\circ} \mathrm{C} \text { (wt. } \%\right)\end{array}$ \\
\hline CTC & 77 & -23 & 1.60 & 2.4 & 11.4 \\
$p$-Xylene & 138 & 13 & 0.861 & 2.3 & 0.568 \\
Toluene & 110 & -95 & 0.866 & 2.4 & 1.37 \\
DCB & 180 & -18 & 1.31 & 9.9 & 9.52 \\
THF & 65 & -108 & 0.888 & 7.5 & 2.26 \\
Chloroform & 61 & -64 & 1.49 & 4.8 & 10.3 \\
TFMB & 102 & -29 & 1.20 & - & n.d. ${ }^{\text {a }}$ \\
MCH & 101 & -126 & 0.77 & - & n.d. ${ }^{\text {a }}$ \\
\hline \hline
\end{tabular}

${ }^{a}$ n.d.: Not dissolved

TFMB dramatically increased the drain current. The hole field-effect mobility $\left(\mu_{\mathrm{FET}}\right)$ of each TFT was determined from the following equation: ${ }^{15}$

$$
I_{\mathrm{D}, \mathrm{sat}}=\frac{W}{2 L} C_{\mathrm{i}} \mu_{\mathrm{FET}}\left(V_{\mathrm{G}}-V_{\mathrm{T}}\right)^{2},
$$

where $I_{\mathrm{D}, \text { sat }}, W, L, C_{\mathrm{i}}$, and $V_{\mathrm{T}}$ are the saturation drain current, channel width, channel length, specific capacitance of the gate dielectric insulator, and threshold voltage, respectively. The hole mobilities were $7.4 \times 10^{-3}$ and $4.8 \times 10^{-2} \mathrm{~cm}^{2} \mathrm{~V}^{-1} \mathrm{~s}^{-1}$ for TFTs prepared without and with TFMB, respectively;
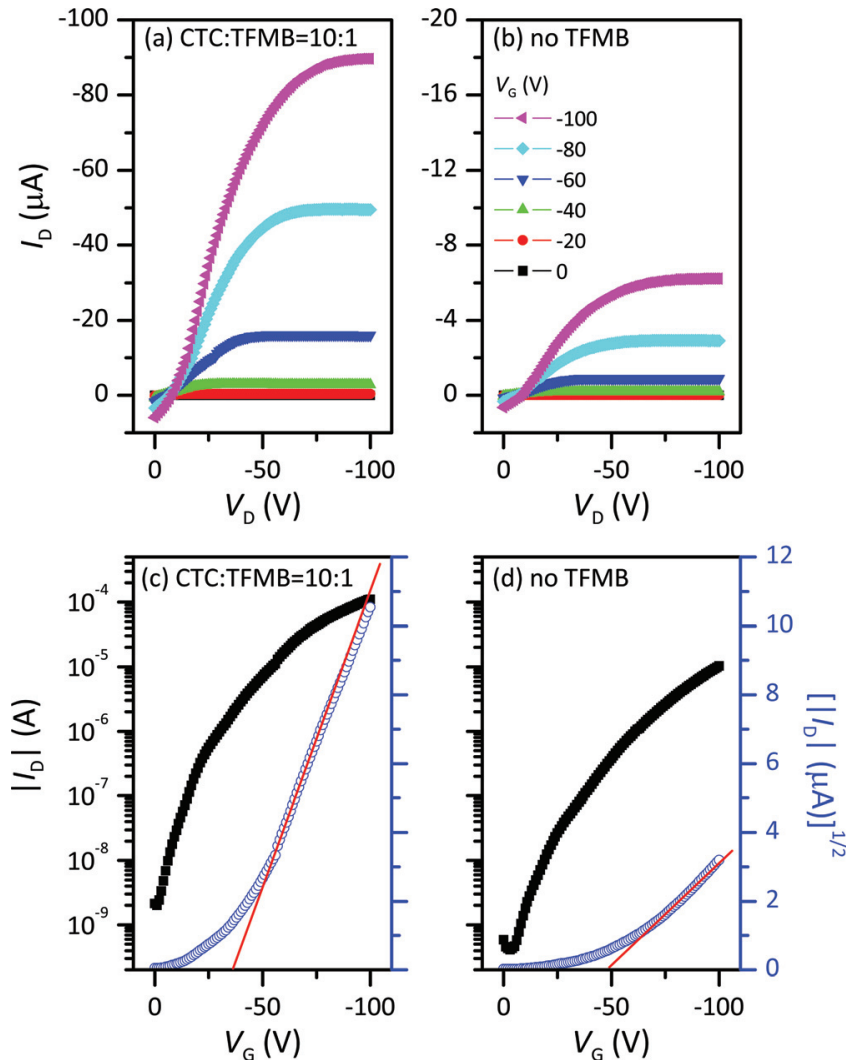

FIG. 2. (Color online) $I_{\mathrm{D}}-V_{\mathrm{D}}$ (a), (b) and $I_{\mathrm{D}}-V_{\mathrm{G}}$ (c), (d) characteristics of P3HT TFTs prepared from solutions of P3HT in CTC with (a), (c) and without (b), (d) TFMB. $I_{\mathrm{D}}-V_{\mathrm{G}}$ curves were measured at a $V_{\mathrm{D}}$ of $-100 \mathrm{~V}$. The legend for (a) is the same as that for (b). 
thus, TFMB addition increased the hole mobility by almost seven times. Figures 2(c) and 2(d) show that the threshold voltage and subthreshold slope (SS) also improved upon TFMB addition. $V_{\mathrm{T}}$ improved from -52.5 to $-36.0 \mathrm{~V}$ and the $\mathrm{SS}$ improved from -10.9 to $-8.0 \mathrm{~V} /$ decade upon adding TFMB.

Figure 3 shows $\mu_{\mathrm{FET}}$ for several individual TFTs prepared from a solution of $\mathrm{P} 3 \mathrm{HT}$ in CTC containing two different concentrations of TFMB; the addition of TFMB enhanced the mobility in P3HT TFTs. Table II summarizes the transistor parameters of P3HT TFTs prepared with several TFMB concentrations. Increasing the CTC:TFMB ratio up to 10:1 led to higher mobility and improved other TFT parameters. Further addition of TFMB slightly reduced the mobility while the other TFT parameters continued to improve.

Figure 4 shows topographical images of P3HT thin films prepared from CTC solutions containing different concentrations of TFMB. The surface morphology dramatically changed depending on the concentration of TFMB. The surface of the film prepared without TFMB was fairly flat with some minute structures. In contrast, the surface of the film prepared with 10:1 CTC:TFMB [Fig. 4(c)] consisted of individual $\sim 100$-nm grains. The mobility in polycrystalline semiconducting layers in TFTs strongly depends on the grain size. ${ }^{16,17}$ Thus the larger grains probably caused the higher mobility in the films formed with a higher concentration of TFMB. The surface of a P3HT thin film prepared from a solution containing 100:1 CTC:TFMB [Fig. 4(b)] is slightly rougher than the thin film prepared without TFMB, corresponding to the moderate mobility in this film.

Figure 5(a) shows XRD patterns for the P3HT thin films prepared from the solutions of CTC containing different concentrations of TFMB. A weak reflection from ITO (211) was observed at a $2 \theta$ of $21.5^{\circ}$ because P3HT TFTs were used as XRD samples. Adding TFMB strongly increased the intensity of the (100) reflection of P3HT, which corresponds to a lamellar structure of P3HT with interdigitated hexyl chains [Fig. 5(b)]. ${ }^{18}$ The more intense (100) peak obtained from the TFTs formed with higher TFMB concentrations indicates that increasing the TFMB concentration increases the propensity for P3HT to adopt an edge-on orientation, where the

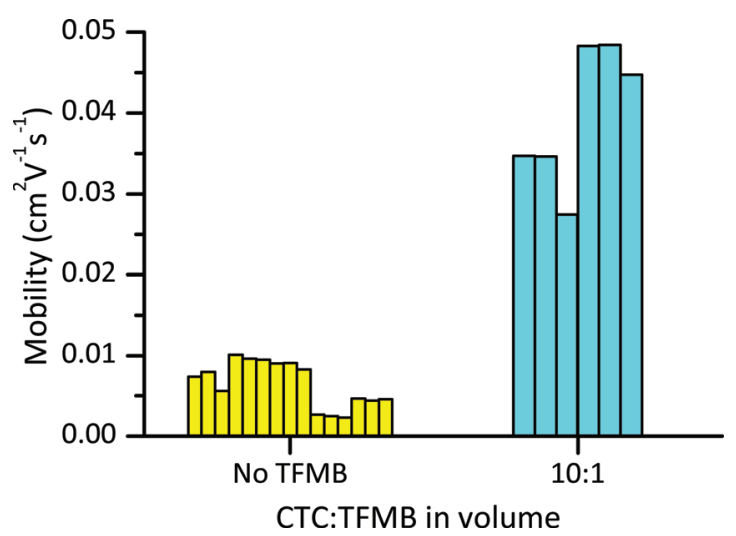

FIG. 3. (Color online) Histograms of the field-effect mobilities of P3HT TFTs prepared from the solutions of P3HT in CTC without (left) and with (right) TFMB.
TABLE II. Parameters of P3HT TFTs prepared from solutions of P3HT in CTC containing various concentrations of TFMB.

\begin{tabular}{lccccc}
\hline \hline $\begin{array}{l}\text { CTC: } \\
\text { TFMB }\end{array}$ & $\begin{array}{c}\mu_{\mathrm{FET}}\left(10^{-2} \mathrm{~cm}^{2}\right. \\
\left.\mathrm{V}^{-1} \mathrm{~s}^{-1}\right)\end{array}$ & $V_{\mathrm{T}}(\mathrm{V})$ & $\begin{array}{c}\text { On/off } \\
\text { ratio }\left(10^{5}\right)\end{array}$ & $\begin{array}{c}\mathrm{SS}(\mathrm{V} / \\
\text { decade) }\end{array}$ & $\begin{array}{c}\text { No. of } \\
\text { samples }\end{array}$ \\
\hline No TFMB & $0.651 \pm 0.282$ & $-48.8 \pm 2.6$ & $0.18 \pm 0.16$ & $12.5 \pm 2.9$ & 15 \\
$100: 1$ & $1.72 \pm 0.48$ & $-52.6 \pm 1.5$ & $0.13 \pm 0.03$ & $11.7 \pm 0.9$ & 12 \\
$50: 1$ & $3.25 \pm 0.50$ & $-47.3 \pm 2.2$ & $1.8 \pm 2.7$ & $8.1 \pm 2.7$ & 4 \\
$10: 1$ & $3.97 \pm 0.87$ & $-35.7 \pm 3.7$ & $1.0 \pm 0.7$ & $6.8 \pm 1.8$ & 6 \\
$5: 1$ & $2.66 \pm 0.57$ & $-34.4 \pm 3.3$ & $1.6 \pm 1.0$ & $3.8 \pm 0.5$ & 10 \\
\hline \hline
\end{tabular}

$a$ axis is normal to the substrate, in the thin films. In contrast, a weak, broad reflection was observed at a $2 \theta$ of around $23^{\circ}$ for the sample prepared without TFMB. This is consistent with the (020) reflection that corresponds to an interchain spacing in P3HT associated with face-to-face packing of the thiophene rings. ${ }^{19-21}$ This reflection indicates that some regions of this P3HT film have adopted a face-on orientation, which has an inferior charge transporting ability compared with that in regions with an edge-on orientation. This broad peak mostly disappeared in the P3HT thin films prepared with TFMB. The increase in the intensity of the (100) peak and disappearance of the (020) reflection peak upon TFMB addition suggests that its addition changed the structures in the P3HT thin films in a manner that facilitated lateral charge carrier transportation. ${ }^{5,22}$ These XRD results are consistent with the performance of TFTs.

In addition, the full width at half maximum intensity $(\beta)$ of the (100) reflection peak decreased slightly as the concentration of TFMB increased, from $\beta=0.68^{\circ}$ for no TFMB to $\beta=0.58^{\circ}$ for 10:1 CTC:TFMB. The large grains observed in Fig. 4(c) are probably too large to explain the smaller $\beta$ for the film prepared using 10:1 CTC:TFMB from the viewpoint of Scherrer's equation: ${ }^{23}$

$$
\tau=\frac{K \lambda}{\beta \cos \theta},
$$

where $K$ is the shape factor, $\lambda$ is the $\mathrm{x}$-ray wavelength, and $\tau$ is the mean size of the ordered (crystalline) domains. Thus the decrease in $\beta$ induced by TFMB addition resulted from the improved quality of the lamellar structure of P3HT inside the grains. We suggest that the improved quality of the grains also caused the enhanced TFT performance. Note that so far all our results indicate that the P3HT thin films have been improved, suggesting that these improvements enhanced the TFT performance. However, it is also well known that the contact resistance of source and drain electrodes with the channel semiconductor affects the TFT performance. In this study, the improvements in the thin films occurred in almost all parts of the films; thus, improving the quality of the semiconducting channels (and not the contact resistance) probably caused the TFT performance enhancements we observed.

\section{B. Effect of TFMB addition on P3HT films formed using other solvents}

The preceding section clearly demonstrated that adding TFMB to the solutions of P3HT in CTC improved the 
(a)

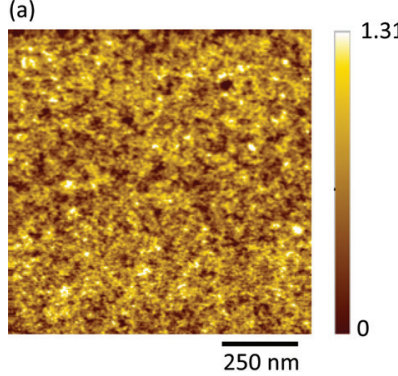

(b)

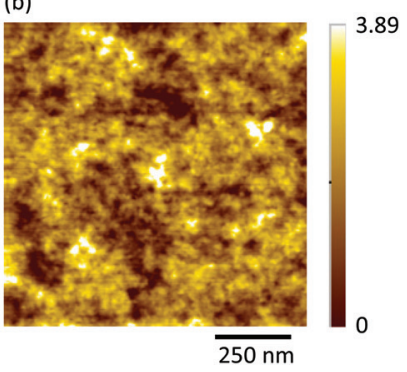

(c)

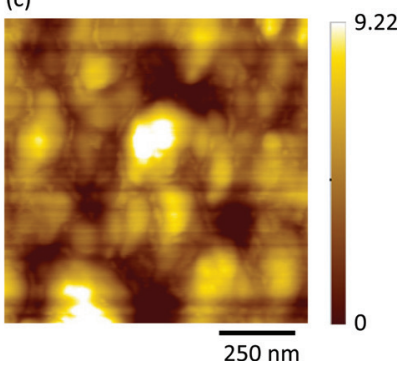

FIG. 4. (Color online) Topographical images of P3HT thin films prepared from solutions of P3HT in CTC containing: (a) no TFMB, (b) 100:1 CTC:TFMB, and (c) 10:1 CTC:TFMB. The color bars next to each image represent the height scale in nanometers. performance of the resulting TFTs. Adding TFMB changed the morphology of the thin films in a way that facilitated charge carrier transportation. The effect may be clear, but the mechanism for the change in morphology is not. To determine this mechanism, we investigated the effect of TFMB addition in various solvents.

Figure 6 summarizes the effect of TFMB addition on the solutions of P3HT in various solvents on the mobilities of TFTs prepared from these solutions. The solvents used in this study can be divided into two categories depending on whether the addition of TFMB effectively changed the mobility. In the first category containing CTC, chloroform, and THF, TFMB addition improved the mobility to various degrees. In contrast, in the second category containing $p$-xylene, DCB, and toluene, the addition of TFMB did not cause a clear change in the mobility. Table I shows that the solvents in the first category have a lower boiling point $\left(T_{\mathrm{b}}\right)$ than that of TFMB, whereas the solvents in the second category have a higher $T_{\mathrm{b}}$ than that of TFMB. Note that no other relationship between a solvent parameter and the TFMBinduced TFT performance improvement was observed.

The preceding findings help in elucidating the mechanism of TFMB influence in some systems. During the deposition of a P3HT layer from solution onto a substrate, the solvent vaporizes. In case of mixed solvents, the composition of the solvent remaining in a developing film varies during the deposition. The solvent with the highest $T_{\mathrm{b}}$ in a mixed solvent system should remain in the developing film during the final stage of deposition. TFMB vaporizes before $p$-xylene, $\mathrm{DCB}$, and toluene because of its lower $T_{\mathrm{b}}$; this explains why TFMB has no clear influence on the TFT performance when these solvents are used. Figure 7 shows AFM images of P3HT thin films prepared from the solutions
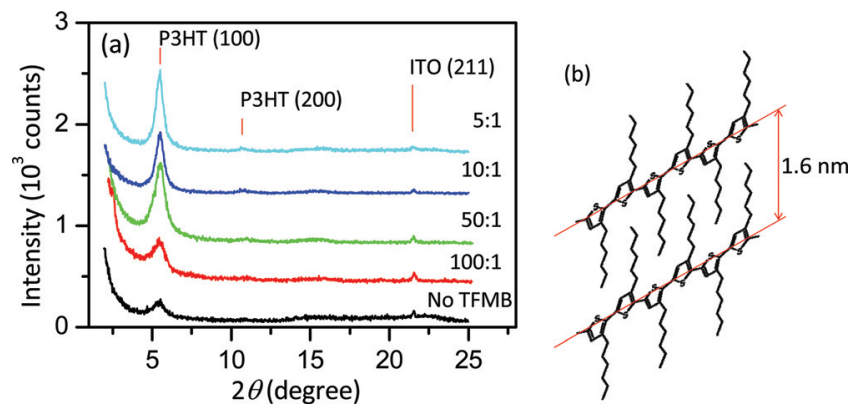

FIG. 5. (Color online) (a) XRD patterns of P3HT TFTs prepared from solutions of P3HT in CTC containing various concentrations of TFMB. (b) Schematic illustration of an interdigitating lamellar structure of P3HT. of P3HT in $p$-xylene or toluene with or without TFMB. The addition of TFMB to the solutions of P3HT in $p$-xylene or toluene did not induce significant morphological changes. Small grains were found over the entire surface with some contacting each other to form fibrillar structures in the P3HT thin film prepared from $p$-xylene with or without TFMB [Figs. 7(a) and 7(b), respectively].

In contrast, the addition of TFMB improved the mobilities of P3HT TFTs formed using CTC, chloroform, and THF. In these cases, TFMB remains in the developing film because of its higher $T_{\mathrm{b}}$. The remaining TFMB probably influences the organization of the developing thin films during the final stage of the drying process, improving the charge carrier transportation in the resulting films. Because $\mathrm{P} 3 \mathrm{HT}$ is insoluble in TFMB, the influence is not based on dissolution; it is more likely that the influence of TFMB arises from precipitation. Here the improvement factor (IF) is defined as the value obtained by dividing the average mobility found for the optimal addition of TFMB by the average mobility with no added TFMB. IFs of 6.1, 2.8, and 2.6 were obtained for CTC, chloroform, and THF, respectively. According to Table I, better solvents for P3HT showed higher IFs. A possible mechanism based on this finding is presented in the next section.

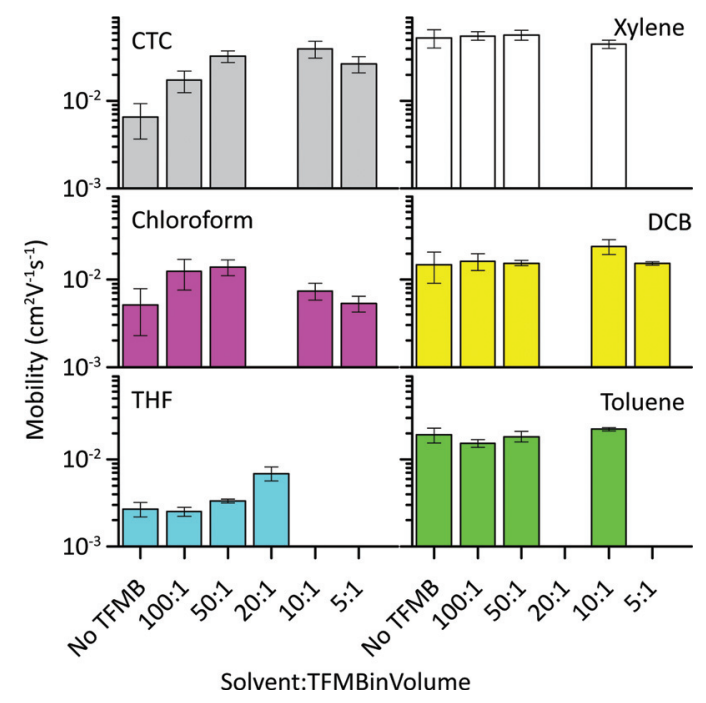

FIG. 6. (Color online) Mobility dependence on TFMB concentration for P3HT TFTs prepared from various solvents. The solubility of P3HT in THF is so poor that a proper thin film for a TFT with a THF/TFMB ratio of 10:1 could not be prepared, so a ratio of 20:1 was used instead. 
(a) Xylene (no TFMB)

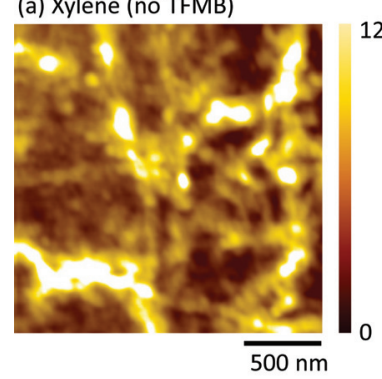

(c) Toluene (no TFMB)

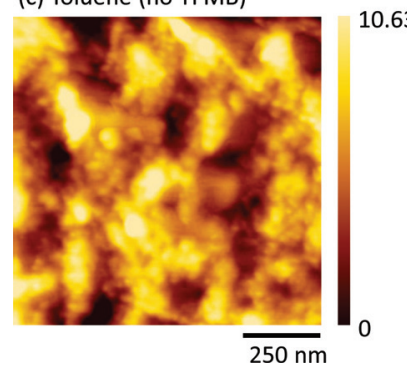

(b) Xylene:TFMB $=10: 1$

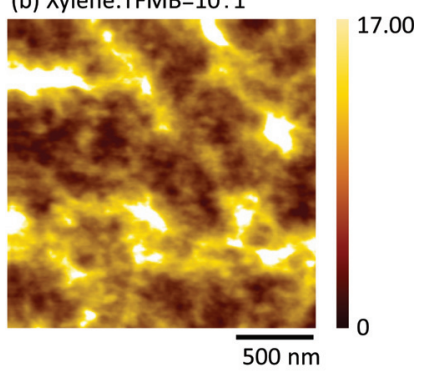

(d) Toluene:TFMB=10:1

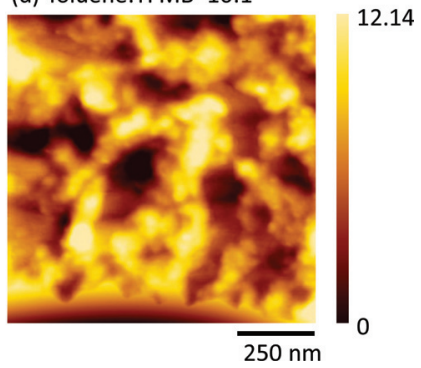

FIG. 7. (Color online) Topographical images of P3HT thin films prepared from solutions of P3HT in p-xylene (a), (b) or toluene (c), (d) without (a), (c) or with (b), (d) TFMB. The color bars next to each image represent the height scale in nanometers.

Note that P3HT TFTs fabricated from $p$-xylene solution exhibited slightly better performance $\left[\mu_{\mathrm{FET}}=(5.30 \pm 1.25)\right.$ $\left.\times 10^{-2} \mathrm{~cm}^{2} \mathrm{~V}^{-1} \mathrm{~s}^{-1}\right]$ than that $\left[\mu_{\mathrm{FET}}=(3.97 \pm 0.87) \times 10^{-2} \mathrm{~cm}^{2}\right.$ $\left.\mathrm{V}^{-1} \mathrm{~s}^{-1}\right]$ of the devices prepared from the solution of P3HT in 10:1 CTC:TFMB. $T_{\mathrm{b}}$ of $p$-xylene is $138^{\circ} \mathrm{C}$, and this high $T_{\mathrm{b}}$ probably had a positive influence on the resulting P3HT thin films as reported by Chang et al. ${ }^{9}$ In fact, as shown in Fig. 5, a narrower (001) reflection compared with the case of the CTC system with no TFMB and the disappearance of the broad (020) reflection around $2 \theta=23^{\circ}$ were observed in the XRD pattern of the P3HT thin film prepared from $p$-xylene solution with no TFMB. These XRD features probably result from the high $T_{\mathrm{b}}$ of xylene, as reported by Chang et al., and they imply that the film possesses a better mobility. However, the P3HT thin film prepared from the CTC solution containing TFMB at the best concentration had a preferable thin-film structure as described in this section. Thus one possible reason for the inferior mobility of the CTC/TFMB-based P3HT TFTs compared to the pristine xylene-based TFTs may be the boundaries of the self-organized crystalline grains.

\section{Mechanism of TFT improvement caused by TFMB addition}

Figure 8(a) schematically depicts the residual volumes of each solvent in a mixed system containing CTC and TFMB during film deposition from solution. CTC vaporizes faster than TFMB because of the difference in their boiling points. Thus the volume ratio of CTC/TFMB gradually changes during film deposition, causing $\mathrm{P} 3 \mathrm{HT}$ to precipitate faster than that in the case of CTC alone because of the insolubility of P3HT in TFMB. Figure 8(b) illustrates the precipitated P3HT aggregates (crystallites). TFMB is a poor solvent for P3HT and stimulates faster precipitation; however, some CTC, which is a good solvent for P3HT, will be incorporated in the aggregates because of the high affinity between CTC
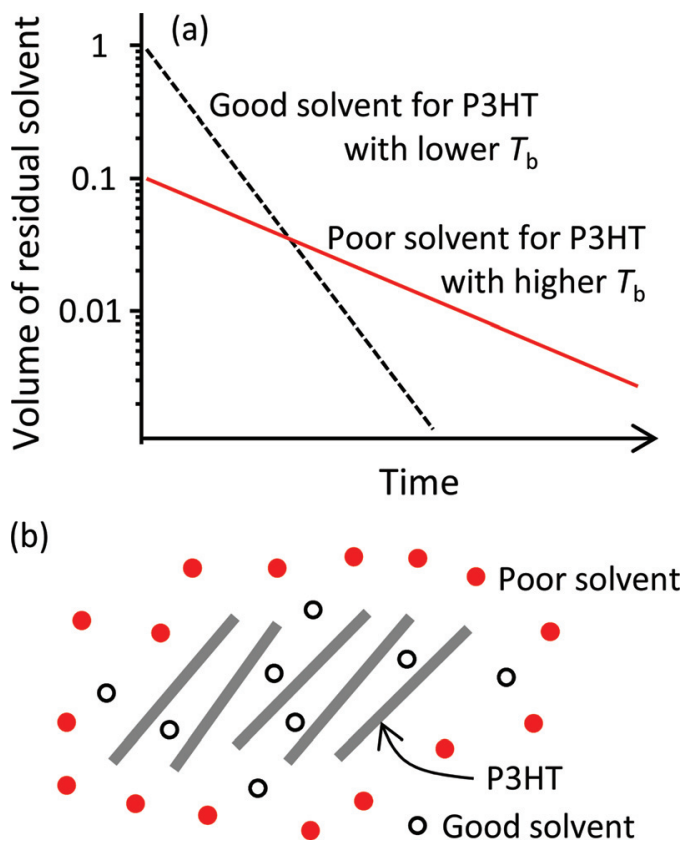

FIG. 8. (Color online) (a) Schematic plot of the time evolution of residual solvents present when depositing a thin film using a mixed solvent system. (b) Schematic illustration of a precipitated aggregate of P3HT in a mixed solvent system.

and P3HT. The incorporated CTC molecules probably work as a plasticizer, allowing the disordered P3HT segments to align better. These CTC molecules then evaporate, and the remaining TFMB molecules cause the aggregates to pack tightly because of the poor solubility of P3HT in TFMB. ${ }^{24,25}$ During these processes, the P3HT aggregates might grow upward and/or congregate together to reduce the unpreferred contact between P3HT segments and TFMB. ${ }^{24}$ The surface morphology of the P3HT thin film prepared from the mixed solution of CTC:TFMB $=10: 1$ [Fig. 4(c)], which shows large round grains, endorses this speculation.

The abovementioned mechanism depends on only $T_{\mathrm{b}}$ and the solubility of P3HT in the solvent. To confirm this hypothesis, TFMB was substituted with $\mathrm{MCH}$, which has a $T_{\mathrm{b}}$ similar to that of TFMB $\left(101^{\circ} \mathrm{C}\right)$ but significantly different electronic interaction characteristics because $\mathrm{MCH}$ is a saturated hydrocarbon. Figure 9 shows that the addition of $\mathrm{MCH}$ to the solutions of P3HT in CTC affected the mobilities of fabricated TFTs. The mobilities changed depending on the concentrations of $\mathrm{MCH}$, and the tendency was very similar to that for the case of TFMB as shown in the figure. This result is consistent with the proposed mechanism.

\section{SUMMARY}

The addition of TFMB or MCH to a solution of P3HT improved the performance of the resulting P3HT TFTs when the solvent had a lower boiling point than TFMB or MCH. The effect was greatest when CTC was used as the solvent. The maximum mobility achieved was $(4.0 \pm 0.9) \times 10^{-2}$ $\mathrm{cm}^{2} \mathrm{~V}^{-1} \mathrm{~s}^{-1}$, which was 6.1 times higher than that of a TFT prepared from a solution without TFMB. The addition of TFMB or MCH also improved the on/off ratio and the subthreshold slope of the resulting TFTs. The boiling point was 
the most important factor affecting the remaining TFMB or $\mathrm{MCH}$ at the final stage of thin film deposition. The poor solubility of $\mathrm{P} 3 \mathrm{HT}$ in this remaining TFMB or $\mathrm{MCH}$ probably influenced the precipitation of $\mathrm{P} 3 \mathrm{HT}$, causing aggregation and/or growth of P3HT precipitates. A small amount of the more volatile solvent is thought to be incorporated into the precipitates, working as a plasticizer and improving their crystallinity.

\section{ACKNOWLEDGMENTS}

This work was supported by the Japan Society for the Promotion of Science (JSPS) KAKENHI (20550162) and the Regional Innovation Cluster Program of Nagano, from MEXT, Japan. This article is dedicated to the first principal of the Faculty of Textile Science and Technology, Shinshu University, Chotaro Harizuka, on the occasion of the 100th anniversary of the faculty.

${ }^{1}$ S. Lee, B. Koo, J. Shin, E. Lee, H. Park, and H. Kim, Appl. Phys. Lett. 88, 162109 (2006).

${ }^{2}$ T. Yamamoto and K. Takimiya, J. Am. Chem. Soc. 129(8), 2224 (2007).

${ }^{3}$ S. Tatemichi, M. Ichikawa, T. Koyama, and Y. Taniguchi, Appl. Phys. Lett. 89(11), 112108 (2006).

${ }^{4}$ T. D. Anthopoulos, B. Singh, N. Marjanovic, N. S. Sariciftci, A. Montaigne Ramil, H. Sitter, M. Colle, and D. M. de Leeuw, Appl. Phys. Lett. 89(21), 213504/213501 (2006).

${ }^{5}$ H. Sirringhaus, P. J. Brown, R. H. Friend, M. M. Nielsen, K. Bechgaard, B. M. W. Langeveld-Voss, A. J. H. Spiering, R. A. J. Janssen, E. W. Meijer, P. Herwig, and D. M. de Leeuw, Nature 401(6754), 685 (1999).

${ }^{6}$ G. Sauve, A. E. Javier, R. Zhang, J. Liu, S. A. Sydlik, T. Kowalewski and R. D. McCullough, J. Mater. Chem. 20(16), 3195 (2010).
${ }^{7}$ Y. Kim, S. Cook, S. M. Tuladhar, S. A. Choulis, J. Nelson, J. R. Durrant, D. D. C. Bradley, M. Giles, I. McCulloch, C.-S. Ha, and M. Ree, Nat. Mater. 5(3), 197 (2006).

${ }^{8}$ S. Cho, K. Lee, J. Yuen, G. M. Wang, D. Moses, A. J. Heeger, M. Surin, and R. Lazzaroni, J. Appl. Phys. 100(11), 114503 (2006).

${ }^{9}$ J. F. Chang, B. Sun, D. W. Breiby, M. M. Nielsen, T. I. Solling, M. Giles, I. McCulloch, and H. Sirringhaus, Chem. Mater. 16(23), 4772 (2004).

${ }^{10}$ S. Hoshino, M. Yoshida, S. Uemura, T. Kodzasa, and T. Kamata, J. Photopolym. Sci. Tech. 17(2), 327 (2004).

${ }^{11}$ B. S. Ong, Y. Wu, P. Liu, and S. Gardner, Adv. Mater. 17(9), 1141 (2005).

${ }^{12}$ C. Aleman and L. Julia, J. Phys. Chem. 100(35), 14661 (1996).

${ }^{13}$ M. Urien, G. Wantz, E. Cloutet, L. Hirsch, P. Tardy, L. Vignau, H. Cramail, and J.-P. Parneix, Org. Electron. 8(6), 727 (2007).

${ }^{14}$ M. Ichikawa, L. You, S. Tatemichi, T. Koyama, and Y. Taniguchi, Jpn. J. Appl. Phys. 45(44), L1171 (2006).

${ }^{15}$ S. M. Sze, Physics of Semiconductor Devices, 2nd ed. (Wiley, New York, 1981).

${ }^{16}$ M. Shtein, J. Mapel, J. B. Benziger, and S. R. Forrest, Appl. Phys. Lett. 81(2), 268 (2002).

${ }^{17}$ A. Di Carlo, F. Piacenza, A. Bolognesi, B. Stadlober, and H. Maresch, Appl. Phys. Lett. 86(26), 263501 (2005).

${ }^{18}$ R. D. McCullough, Adv. Mater. 10(2), 93 (1998).

${ }^{19}$ T.-A. Chen, X. Wu, and R. D. Rieke, J. Am. Chem. Soc. 117(1), 233 (1995).

${ }^{20}$ T. J. Prosa, M. J. Winokur, and R. D. McCullough, Macromolecules 29(10), 3654 (1996).

${ }^{21}$ W. Ma, C. Yang, X. Gong, K. Lee, and A. J. Heeger, Adv. Funct. Mater. 15(10), 1617 (2005).

${ }^{22}$ D.-H. Kim, Y.-D. Park, Y. Jang, H. Yang, Y.-H. Kim, J. I. Han, D. G. Moon, S. Park, T. Chang, C. Chang, M. Joo, C.-Y. Ryu, and K. Cho, Adv. Funct. Mater. 15(1), 77 (2005).

${ }^{23}$ B. D. Cullity and S. R. Stock, Elements of X-Ray Diffraction, 3rd ed. (Prentice-Hall, New York, 2001).

${ }^{24}$ M. Doi and S. F. Edwards, The Theory of Polymer Dynamics, 1st ed. (Oxford University Press, Oxford, UK, 1986).

${ }^{25}$ A. Montesi, M. Pasquali, and F. C. MacKintosh, Phys. Rev. E 69(2), 021916 (2004). 